\title{
Une nouvelle approche pour la protection des plages : Application à la plage du lido de Sète Damien ISEBE $^{1}$, Frédéric BOUCHETTE ${ }^{1,2}$, Bijan MOHAMMADI ${ }^{1}$, Pascal AZERAD ${ }^{1}$, Adrien LAMBERT ${ }^{2}$, Nans BUJAN ${ }^{2}$, Florent GRASSO ${ }^{3}$, Hervé MICHALLET ${ }^{3}$
}

\author{
${ }^{1}$ Université Montpellier 2, Institut de Mathématiques et de Modélisation de \\ Montpellier, UMR 5149 - C.C. 051, \\ Place Eugène Bataillon, 34095, Montpellier, France \\ damien.isebe@univ-montp2.fr \\ ${ }^{2}$ Université Montpellier 2, Geosciences Montpellier, UMR 5243 - C.C. 60 , \\ Place Eugène Bataillon, 34095, Montpellier, France \\ bouchette@gm.univ-montp2.fr \\ ${ }^{3}$ LEGI - Domaine Universitaire BP 53 \\ 38041 Grenoble Cedex 9, France
}

\section{Résumé :}

Des techniques d'optimisation de formes sont utilisées dans des problèmes du Génie Côtier pour la protection des plages. En collaboration avec le bureau d'étude Bas-Rhône Languedoc ingénierie, un projet d'aménagement concret de la plage du Lido de Sète, utilisant des ouvrages immergés en géotextile, a été étudié. Des solutions optimales, visant à réduire la mise en suspension des sédiments, pour les structures de défenses ont été obtenues à partir d'un modèle hydrodynamique de propagation de houle. Une étude complémentaire à partir d'un modèle de circulation et des travaux expérimentaux en canal à houle permettent ensuite de valider les résultats optimaux numériques obtenus.

\section{Abstract :}

Shape optimization techniques, focusing on coastal structures design, are used to solve coastal engineering problem. In collaboration with the Bas-Rhône Languedoc engineering Company, a actual development plan concerning the beach of the Lido of Sète has been studied. Optimal solutions, aimed at reducing sediments suspended, for defense structures have been obtained with a hydrodynamic water wave model. An additional study using a circulation model and experimental works in flume tend to validate optimal numerical results.

Mots-clés :

Génie Côtier - Optimisation de formes - Hydrodynamique littorale - Impact sur 
ouvrages côtiers - Circulation littorale - Acquisition données expérimentales

\section{Introduction}

L'érosion des plages s'est amplifié, de manière parfois catastrophique, depuis une vingtaine d'années à tel point que de nos jours, sur l'ensemble de la planète, $70 \%$ des plages reculent alors que seulement $10 \%$ ont tendance à avancer (PASKOFF, 2006). Pour lutter contre ce phénomène, les principales techniques de défenses utilisées sont la mise en place d'ouvrages d'art tels que épis ou briselames. Cependant, l'efficacité de ces méthodes du Génie Côtier s'avèrent limitée et locale. En effet, après une phase d'amélioration rapide, elles aboutissent souvent à une aggravation de l'érosion des secteurs adjacents, ce qui demande une installation de nouveaux ouvrages d'art (voir Figure 1.).

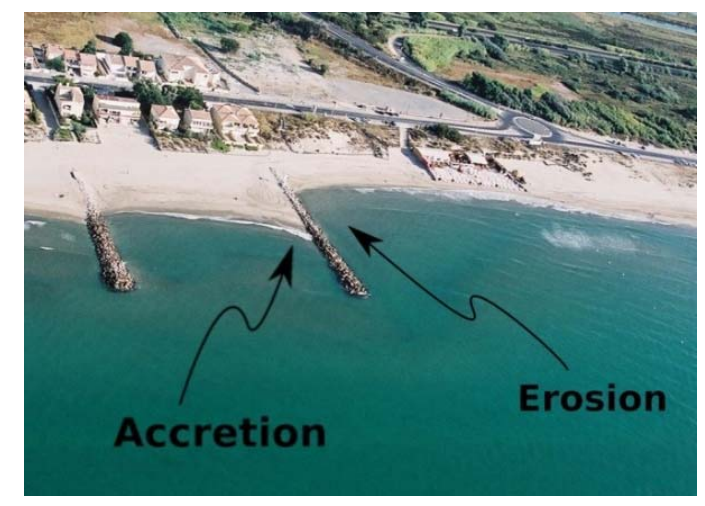

Figure 1. Exemple d'implantation classique d'épis. On aperçoit un mécanisme d'érosion/accrétion autour de l'ouvrage.

Nous présentons une approche novatrice qui consiste à utiliser des techniques d'optimisation de formes portant sur la conception d'ouvrage en mer dans des problèmes du Génie Côtier.

Ces techniques sont appliquées à la protection de la plage du Lido de Sète (Mer Méditerranée, Languedoc-Roussillon, France) dans le cadre d'une collaboration avec le bureau d'étude BRL ingénierie. Il s'agit d'un cas concret de plage soumise à une très forte érosion. Ces travaux font partis du projet ANR COPTER (Conception, Optimisation et ProTotypage d'ouvrages de lutte contre l'ERosion en domaine littoral) ayant pour objectif de développer des outils d'optimisation et d'aide à la conception d'ouvrages de protection des plages afin d'augmenter leur efficacité.

\section{Principe de l'optimisation de formes en Génie Côtier}

Le terme optimisation de formes fait référence à l'ensemble des techniques 
d'optimisation numérique qui permettent la modification progressive de la forme d'un domaine quelconque soumis à des processus physiques, en fonction de critères d'optimisation judicieusement choisis. En d'autres termes, nous cherchons à optimiser des profils/formes d'ouvrages qui favorisent l'accrétion un peu à la manière dont on optimise, en aéronautique, le profil d'une aile d'avion pour augmenter la portance et diminuer la traînée.

Pour ce faire, nous avons utilisé une méthode d'optimisation globale (ISEBE et al., 2006), capable d'atteindre le minimum d'une fonction coût (i.e. le critère d'optimisation) à priori non-convexes pouvant posséder plusieurs minima locaux. Ici, la variable paramètre la forme de la structure et peut-être assimilée à un ensemble de points de contrôle. Pour calculer, il faut donc d'abord calculer la houle dans le domaine, qui lui même dépend de la forme de l'ouvrage, elle-même déterminée et adaptée progressivement par l'algorithme d'optimisation.

\section{Modèle hydrodynamique}

Pour la modélisation de la propagation de la houle vers la côte sur une bathymétrie arbitraire, nous utilisons modèle REF/DIF, basé sur une extension parabolique de l'équation de pente douce de Berkhoff

$$
2 i k C C_{g} \frac{\partial A}{\partial x}+2 k\left(k-k_{0}\right)\left(C C_{g}\right) A+i A \frac{\partial\left(k C C_{g}\right)}{\partial x}+\frac{\partial\left(C C_{g} \frac{\partial A}{\partial y}\right)}{\partial y}-k\left(C C_{g}\right) K^{\prime}|A|^{2} A=0
$$

où $\mathrm{A}$ est l'amplitude complexe de la houle, $\mathrm{k}$ le nombre d'onde, $\mathrm{k} 0$ le nombre d'onde de référence issu des conditions de la houle incidente venant du large, $\mathrm{C}$ la vitesse de phase ( étant la fréquence angulaire de la houle), $\mathrm{Cg}$ la vitesse de groupe, $\mathrm{h}$ la profondeur locale et et $\mathrm{K}^{\prime}$ une fonction de $\mathrm{k}$, $\mathrm{h}$.

Le code numérique, nommé REF/DIF, a été développé au laboratoire Center for Applied Coastal Research (University of Delaware, USA) par J.T. Kirby et R.A. Dalprymple (KIRBY \& DALRYMPLE, 1995). Il calcule la propagation et la transformation d'une houle quelconque sur une topographie modérément irrégulière. En particulier, il gère à la fois la diffraction et la réfraction de la houle sur la topographie, en considérant une forme non-linéaire de l'équation de Berkhoff, modifiée pour tenir compte de la diffraction telle qu'elle a été définie par Yue \& Mei (MEI \& YUE, 1980). Il gère également la dissipation de l'énergie de la houle induite à l'interface eau/air, dans la masse fluide, à l'interface eau/sédiment et par le déferlement. La limitation majeure de ce modèle est qu'il ne gère pas la réflexion. Il doit donc être utilisé sur des domaines à limites dissipatives. Ce modèle, largement validé par la communauté scientifique, est particulièrement bien adapté à l'étude qui suit sur la protection de la plage du Lido de Sète.

\section{Cadre d'étude}




\subsection{Position du problème}

Le Lido de Sète est une barre de terre de $20 \mathrm{~km}$ de long soumise à des houles modérées. Il sépare la mer de l'Etang de Thau. A l'Est, le mont Saint-Clair surplombe le lido, tandis qu'à l'Ouest, on retrouve la côte volcanique d'Agde. Cette configuration restreint les apports/pertes de sable par des courants longshores sur la zone même du lido. Toutefois, le transport de sédiment proche de la côte se fait en direction de l'Ouest et la côte Est du Lido en est directement la source.
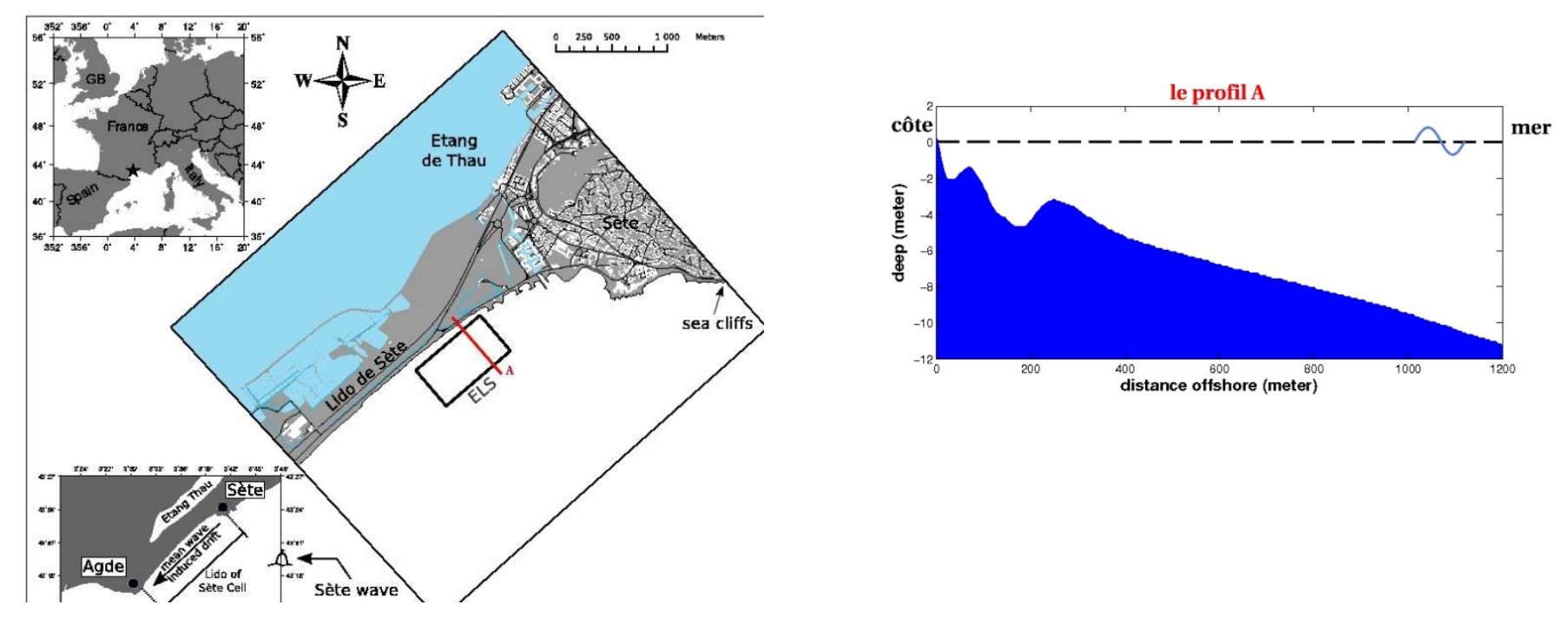

Figure 2. (Gauche) : Localisation de la cellule sédimentaire du Lido de Sète. Le site ELS (Est Lido Sète), le plus touché par l'érosion côtière, représente la zone d'étude. (Droite) : Le profil morphologique moyen de la plage de Sète (ELS). Il s'agit d'un profil typique de plages sableuses comprenant 2 barres sableuses naturelles.

Le long d'un profil cross-shore, la morphologie du site est la suivante: un fond marin sableux classique composé de 2 barres sableuses linéaires, une plage sableuse émergée de 20 à $120 \mathrm{~m}$ de large puis une lagune de 1 kilomètre de large (Etang de Thau) (voir Figure 2.).

Du point de vue de l'érosion, la plage est particulièrement vulnérable lors des tempêtes. En particulier, on peut noter un recul d'environ $50 \mathrm{~m}$ du trait de côte depuis 1967 (1m/an). Actuellement, la ligne de côte a atteint un tel niveau de recul que la route longeant la côte est régulièrement détruite.

Le bureau d'étude $B R L$ ingénierie est porteur du projet de grande envergure 
visant à réaménager la plage du Lido de Sète. Un déploiement sur site de la solution adoptée est prévu pour 2009. BRL ingénierie propose d'utiliser une nouvelle génération d'ouvrages immergés atténuateurs de houle, agissant directement sur la dynamique naturelle de la plage. Il s'agit de tubes en géotextile remplis de sable. La démarche de réaménagement du lido est la suivante (Figure 3.)

$>$ un apport de sable directement sur la plage pour la reconstruire,

$>$ un rechargement en sable des deux barres naturelles dans le but d'augmenter le volume de sable près de la côte,

la disposition d'un ensemble de deux rangées de tubes géotextile immergés disposés côte-à-côte. Pour la modélisation, nous assimilerons les deux tubes géotextile à un seul tube géotextile deux fois plus large.
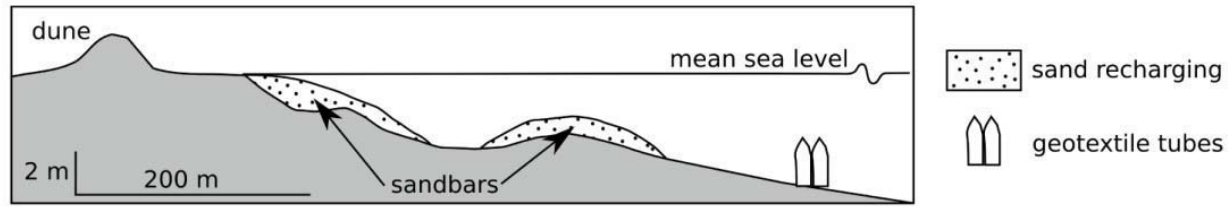

Figure 3. Le projet d'aménagement de la plage du Lido de Sète.

Cette barre artificielle devra donc agir directement sur les houles de tempêtes afin de les atténuer et d'empêcher ainsi l'arrachage de sédiment. Elle doit être initiatrice de la déposition de sédiments à la côte en modifiant les courants et les vitesses orbitales proche de la côte. Leur position et leur dimensionnement reste cependant à définir. Cette étude porte sur la recherche de paramètres optimaux pour la forme et la position de la rangée de tubes géotextiles dans le but de réduire l'énergie responsable de la mise en suspension des sédiments.

\subsection{Les données de houles}

Les données utilisées proviennent du houlographe mouillé au large de Sète (hauteur, période et fréquence d'observation de la houle). Elles ont été récupérées auprès du CETMEF (Centre d'Etudes Techniques Maritimes Et Fluviales) et la base de données CANDHIS (Centre d'Archivage National de Données de Houle In-Situ).

Sur le site de Sète, quatre directions prépondérantes de propagation de houle ont été mises en évidence (Sud, Sus Sud Est, Est Sud Est, Est). De plus, pour chaque direction, les houles observées peuvent être classifiées en 2 catégories : les houles destructrices $(\mathrm{Hs}>2 \mathrm{~m})$ et constructrices $(\mathrm{Hs}<2 \mathrm{~m})$. Les houles constructrices, 
celles qui sont observées le plus souvent sur le site, s'avèrent être bénéfiques à la dynamique naturelle de la plage et doivent être préservées. Les houles destructrices, présentes lors de évènements de tempêtes, sont directement responsables de l'érosion favorisant une remobilisation massive du sable et un export au-delà des barres sableuses d'avant-côte.

\subsection{Les paramètres d'optimisation}

\subsubsection{La paramétrisation d'un tube en géotextile}

L'objectif est de modéliser des tubes en géotextile de manière à contrôler leur forme et position (voir Figure 4.). Pour ce faire, nous utilisons tout d'abord des points de contrôle permettant de déterminer la position et la forme de l'ouvrage dans le domaine ( 2 points de contrôle permettent d'obtenir un ouvrage rectiligne alors que 3 points de contrôle ou plus, définissent des formes d'ouvrages originales par l'utilisation de splines). Le profil vertical de l'ouvrage est ensuite défini en utilisant une fonction Gaussienne de la forme, ce qui rajoute 2 paramètres supplémentaires contrôlant la hauteur et la largeur de l'ouvrage.

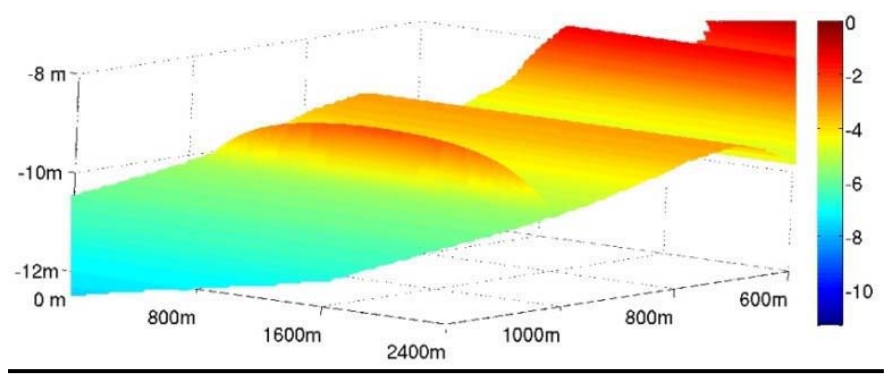

Figure 4. Exemple de modélisation de tube en géotextile sur une topobathymétrie.

\subsubsection{Le critère d'optimisation}

Dans cette étude, on considère qu'un tube en géotextile sera efficace s'il diminue la mise en suspension des sédiments entre les deux barres sableuses naturelles (fosse de lévigation). Ainsi, nous cherchons à déterminer les paramètres optimaux pour les structures qui permettent de diminuer l'énergie potentielle (au sens de la théorie linéaire de la houle) ainsi que la vitesse orbitale des houles destructrices dans la fosse de lévigation.

De plus, l'optimisation est multi-critère car on cherche à trouver le meilleure structure possible pour quatre directions de propagation principales.

\section{5 $\underline{\text { Résultats d'optimisation }}$}

Ce problème de protection de la plage du Lido de Sète est un problème concret 
soumis à plusieurs contraintes industrielles imposées par le bureau d'étude $B R L$ ingénierie (largeur de la structure fixée à 12 mètres, les géotextiles sont parallèles à la côte, tirant-d'eau pour la navigation des bateaux,...). Ainsi, les paramètres à optimiser sont réduits à la distance à la côte et la hauteur de l'ouvrage.

Une étude préliminaire a été réalisée en fixant la hauteur de l'ouvrage à $3 \mathrm{~m}$. Ainsi, en échantillonnant la distance à la côte (avec un pas de $10 \mathrm{~m}$ ), on obtient une courbe montrant l'évolution du critère d'optimisation en fonction de la position de l'ouvrage. On remarque que la fonctionnelle est clairement nonconvexe et qu'il n'est donc pas du tout évident d'assurer l'obtention d'un minimum global en utilisant un algorithme d'optimisation classique. L'utilisation d'un algorithme d'optimisation globale s'avèrent importante pour ce genre de problème industriel. Concernant l'allure de la fonctionnelle, on s'aperçoit que pour une hauteur d'ouvrage de $3 \mathrm{~m}$, la position optimale semble se situer aux alentours de $350 \mathrm{~m}$ de la côte (voir Figure 5.).

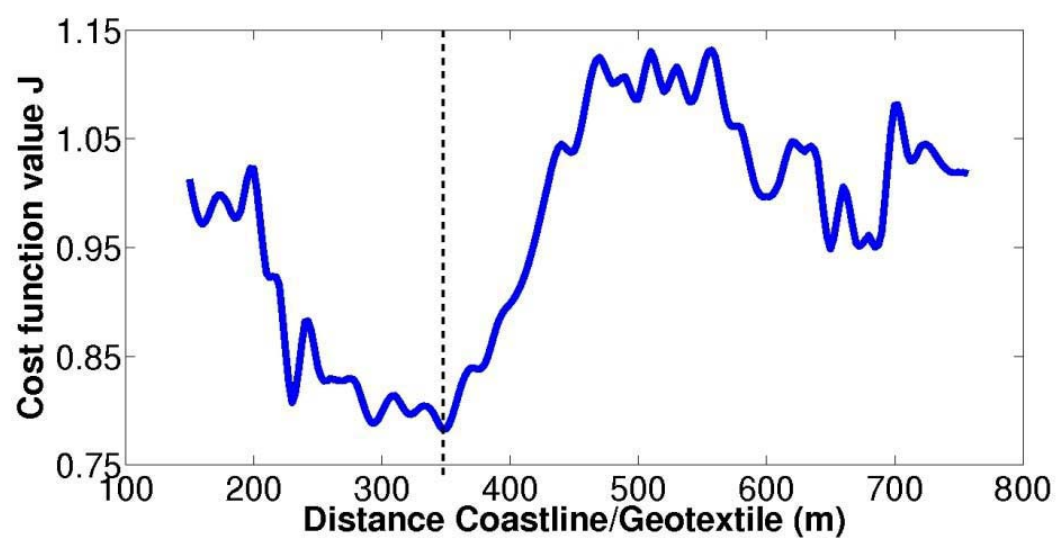

Figure 5. Evolution du critère d'optimisation den fonction de la distance du géotextile à la côte (hauteur du géotextile fixée à 3 mètres.

A partir de cet échantillonnage, nous appliquons ensuite la méthode d'optimisation aux paramètres distance et hauteur du tube géotextile. Nous obtenons la configuration optimale suivante: la rangée de tubes géotextile doit être disposée à $353 \mathrm{~m}$ de la côte et le tube géotextile a une hauteur de $2.5 \mathrm{~m}$. Le tube géotextile optimisé diminue globalement la fonction coût de $24 \%$ (avec un maximum de 30\% pour les houles destructrices de direction Sud Sud Est) (voir Figure 6.). De plus, l'ouvrage est bien transparent vis à vis des houles constructrices ce qui permet de préserver la dynamique naturelle de la plage. 


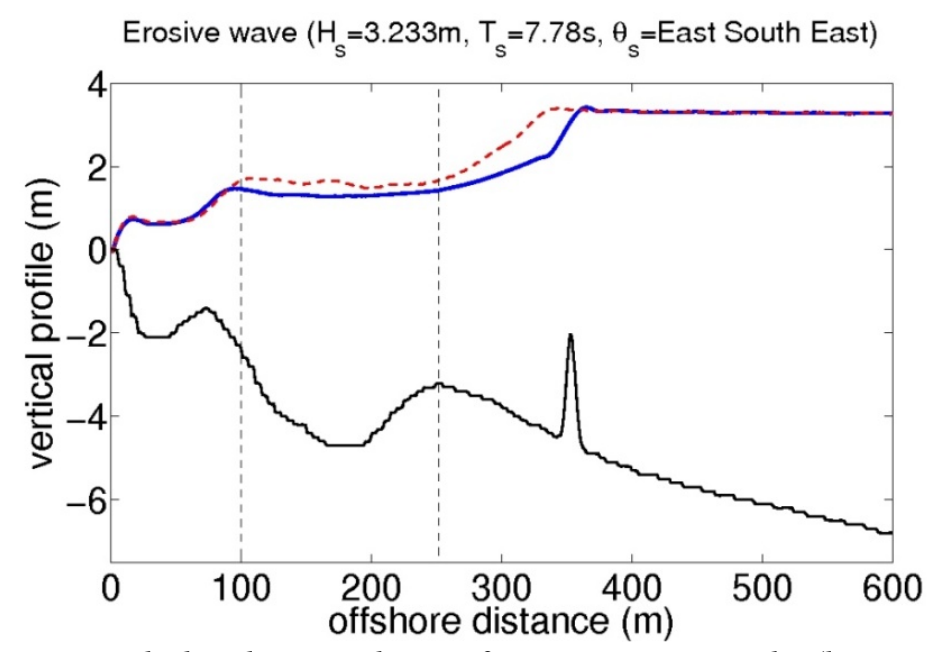

Figure 6. Hauteur de houle pour la configuration optimale (ligne continue) et la configuration initiale (ligne pointillée) dans la cas d'une houle destructrice. On note une diminution de la hauteur de houle dans la fosse de lévigation

Cette étude montre que l'utilisation de techniques d'optimisation de formes permet d'obtenir des solutions opérationnelles pour ce type de problèmes concrets soumis à plusieurs contraintes industrielles (ISEBE et al., 2007).

\section{Discussion et validation expérimentale}

\subsection{Le rôle des courants?}

Les résultats, présentés dans la section 5 , sont issus du couplage entre une méthode d'optimisation et le modèle hydrodynamique REF/DIF de réfractiondiffraction. Ainsi, lorsque nous avons établi le critère d'optimisation (réduction de la mise en suspension du sédiment), nous avons uniquement pris en compte des processus physiques pertinents donnés par le modèle (énergie potentielle de la houle, vitesse orbitale). Cependant, d'autres processus (non pris en compte dans notre critère d'optimisation car non représentés par le modèle) tiennent un rôle important dans le phénomène d'érosion littorale. En particulier, les courants de circulations à l'arrière de l'ouvrage optimisé (principalement dans la fosse de lévigation) peuvent favoriser un transport longshore du sédiment, surtout s'ils sont amplifiés par la structure optimale.

Afin de s'assurer que la configuration optimale n'altère pas les courants, nous utilisons le modèle de circulation quasi-3D SHORECIRC (PUTREVU \& SVENDSEN, 1999). Les résultats, présentés dans la figure 7, montrent qu'il n'y a pas de modification significative des courants en terme d'ordre de grandeur et de direction par rapport au Lido non-protégé. Les courants n'étant pas modifiés par la structure optimale alors que la capacité de remise en suspension des sédiments est fortement réduite, on peut donc envisager une réduction globale du transport 
de sédiment longshore pour la configuration optimale.
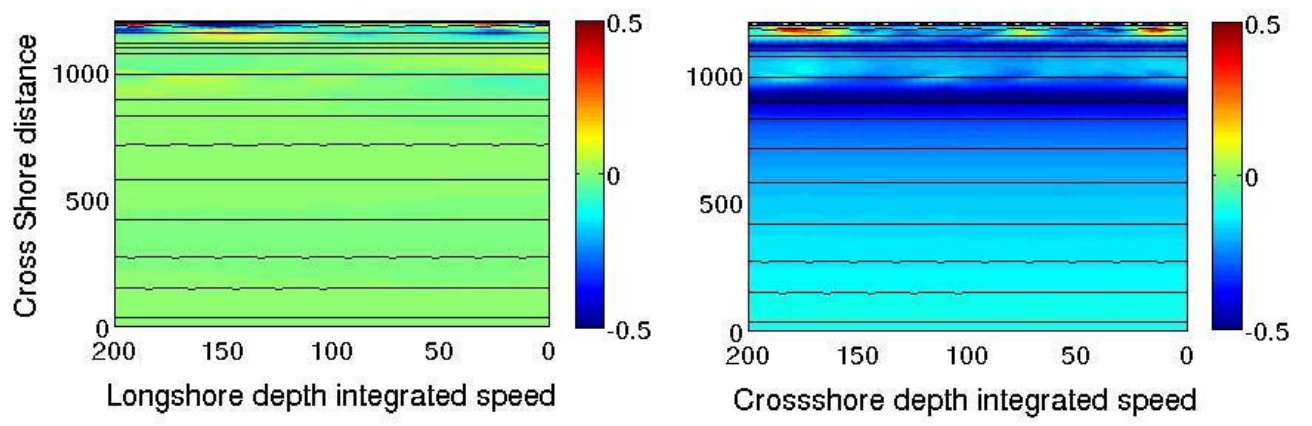

Figure 7. Gauche (resp. Droite): Composante longshore (resp. cross-shore) de la vitesse intégré sur la colonne d'eau.

\subsection{Expériences en canal à houle}

Dans le cadre du projet COPTER et du programme national MODLIT, deux mois d'essais dans le canal à houle $(36 \mathrm{~m}$ sur $56 \mathrm{~cm}$ ) (voir Figure 8.) du LEGI (Grenoble) ont été effectués. L'objectif de ces manips est triple :

1. Valider les résultats numériques d'optimisation obtenus pour la protection d'une plage similaire à celle de Sète (validation des techniques en tant qu'outil d'aide à la décision pour l'aménagement des côtes),

2. Etudier la stabilité des structures artificielles déployées,

3. Traiter la question de la réponse morphodynamique de la plage en configuration optimale.

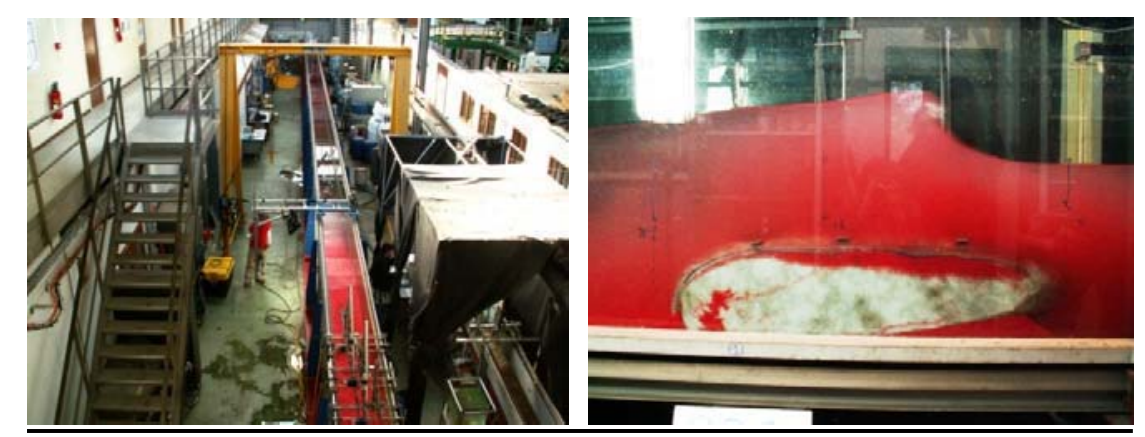

Figure 8. Gauche : Vue de haut du canal à houle du LEGI; Droite : Prototypage de l'ouvrage atténuateur de houle dans le canal.

Pour des houles de tempêtes caractéristiques de Sète, les premiers traitements des données (en cours de publication) confirment l'effet de filtre pour la structure artificielle optimisée ainsi que le renforcement de la barre sableuse naturelle par la structure. On ne note également pas de déformation significative du trait de côte.

\section{$7 \quad$ Conclusion}


Nous avons introduit une méthode d'optimisation de formes pour aborder des problèmes de protection des plages en Génie Côtier. Nous avons montré que l'utilisation d'un modèle hydrodynamique permet d'obtenir des solutions opérationnelles pour un problème industriel concret fortement contraint. La validation des résultats numériques par des expériences en canal à houle permet de conforter l'utilisation d'une approche hydrodynamique. Néanmoins, l'objectif maintenant est d'étendre ces techniques à d'autres modèles et d'autres paramétrisations.

\section{$8 \quad$ Remerciements}

Les auteurs tiennent à remercier tous ceux qui ont contribués à la réalisation de ce projet : BRL ingénierie (Nîmes, France), BRGM (Bastia, France), Cléa Denamiel (HR Wallingford, U.K.), NANS Bujan (Geosciences Montpellier-EID), Raphael Certain (LEGEM, Perpignan). Les auteurs remercient également Pierre Yves Valentin de la Direction Régionale de l'Equipement pour les différents documents et données sur le Lido de Sète, ainsi que l'Entente Interdépartementale de Démoustication pour les données topobathymétriques. Enfin, les auteurs remercient le LEGI (Grenoble) pour son implication dans ce projet depuis 2008.

\section{$9 \quad$ Références bibliographiques}

1 CERTAIN R., (2002). Morphodynamique d'une côte sableuse microtidale à barres: le golfe du lion (Languedoc-Roussillon), Thèse, Université de Perpignan. 2 ISEBE D., AZERAD P., BOUCHETTE F., IVORRA B., MOHAMMADI B., (2007). Shape optimization of geotextile tubes for sandy beach protection, Int. J. Num. Meth. Eng. (à paraître).

3 ISEBE D., AZERAD P., BOUCHETTE F., MOHAMMADI B., (2007). Optimal shape design of defense structures for minimizing short wave impact, Coastal Engineering, $\mathrm{n}^{\circ}$ 55(1), pp. 35-46.

4 ISEBE D., IVORRA B., MOHAMMADI B. (2006). Progress in global optimization and shape design, High Performance Scientific Computing, Springer Science, $10 \mathrm{p}$.

5 KIRBY J.T., DALRYMPLE R.A. (1995). REFDIF1 v2.5 Reffraction Diffraction model, Technical Report, CACR, Beach and Shoreface Morphodynamics, Wiley, $380 \mathrm{p}$.

6 MEI C.C., YUE D.K.P (1980). Forward diffraction of Stokes waves by a thin wedge, J. Fluid. Mech., n99(1), pp. 33-52.

7 PASKOFF R. (2006). Les plages vont-elles disparaîtres?. Le Pommier. 8 PUTREVU U., SVENDSEN I.A., (1999), Three-dimensional dispersion of momentum in wave-induced nearshore currents, Eur. J. Mech., n¹8, pp. 409-427 